\title{
IS THERE ANY SCIENTIFIC EVIDENCE FOR THE PREVENTION OF PREECLAMPSIA WITH LOW-DOSE ASPIRIN? META-ANALYSIS VERSUS RANDOMIZED CONTROLLED TRIAL TO ANSWER THIS QUESTION.
}

Rodrigo Ruano, Rosana S. Fontes, and Marcelo Zugaib

Recently we published a systematic review of the main randomized controlled trials that had evaluated the efficacy of low-dose aspirin to prevent preeclampsia. ${ }^{1}$ For this purpose, we decided to divide the population in 2 different groups: patients at low-risk and at high-risk for preeclampsia. In order to scientifically evaluate this problem, we performed a meta-analysis. Our results almost proved our clinical hypotheses as follows: i) low-dose aspirin has no beneficial in the low-risk group but has a very small effect in the high-risk group, and ii) meta-analysis is not the best scientific method to resolve this question, as it can be unreliable when involving special issues.

Our first hypothesis is of course based on our clinical experience, which is based also on the pathophysiological aspects of preeclampsia. In our clinical experience, we have not observed prevention of preeclampsia in patients that have used low-dose aspirin, which is also demonstrated by recent controlled (randomized) trials, with less bias of case selection. ${ }^{3,4}$ These more recent results can be explained by the fact that secondary prevention is based on the pathophysiological concepts of preeclampsia, which have yet to be clarified. For instance, are the relative reductions of vasodilator prostaglandins (protacyclin) and the increase of vasoconstricting prostaglandin (thromboxane) really responsible for the onset of preeclampsia? Here, we thank Pereira et $\mathrm{al}^{4}$ for helping us to answer the first question.

Our second hypothesis, that meta-analysis is not the best scientific method to answer this question, as it is in fact unreliable in a few situations, has now been demonstrated by Pereira et al. ${ }^{4}$ As these authors concluded, "results of a meta-analysis are model-dependent" 5 and "results from meta-analytic studies can be seriously overestimated, yield-

Nutrition Support Service and Hemodialysis Unit ${ }^{1}$, Santa Rita de Cassia Hospital and Faculty of Medical Sciences of Santa Casa - Vitória/ES, Brazil. Hospital das Clínicas ${ }^{2}$, Faculty of Medicine, University of São Paulo - São Paulo/SP, Brazil.

E-mail: faintuch@net.ipen.br

Received for publication on June 03, 2004.

Accepted for publication on February 14, 2005. ing misleading conclusions." ${ }^{\prime 6}$ As we are living in an era of "evidence-based medicine," meta-analysis has become one of the main scientific tools for researchers to arrive at conclusions regarding medicine. However, on the other hand, this type of analysis has become very risky, as many researchers, sitting behind a computer, evaluate data published by other researchers and analyze such results without having adequate clinical experience.

From this point of view, we welcome the re-analysis of our data ${ }^{4}$, which reinforces what we had already shown ${ }^{1}$. Firstly, because Pereira et $\mathrm{al}^{4}$ "found no convincing evidence for a protective effect of low-dose aspirin under this model (DL Common RR $=0.835 ; 95 \% \mathrm{CI}=0.697$ to 1.001 , $P=0.051)$ "; this they characterized as "surprising," since they probably lack adequate clinical experience on the issue, having only conducted a computer analysis of results from other researchers. Secondly, to re-analyze our data, they did not have to go back to each individual study which shows our report contained sufficient information. However, our intention in writing this revision was to stimulate young researchers to maintain a healthy scientific skepticism when analyzing research reports, even those which describe themselves as "controlled randomized studies." To do this, it is essential to evaluate each original paper! When Pereira et $\mathrm{al}^{4}$ re-analyzed our data; they did not directly read the original studies, as is clear from their comments. For example, they mention that although we "correctly tested the heterogeneity among effect sizes of individual studies," we "have not considered adequately this finding." Of course we did: our discussion is almost entirely directed towards this problem. There are 2 main clinical problems in our meta-analysis, which we also pointed out. First is the dose of aspirin, which varied from 60 to $150 \mathrm{mg} /$ day. Second, which is the most important, selection criteria varied greatly in the different studies, and even inside each study. Our test for heterogeneity showed this in low-risk $(P=0.01)$ and high-risk patients $(P=0.06)$. Conducting a re-analysis and applying different statistical methods, Pereira et $\mathrm{al}^{4}$ con- 
firmed this fact observing "significant evidence for heterogeneity both in the asymptotic $\left(\mathrm{c}^{2}=25.86\right.$, df $=16, P=$ 0.056) and in the parametric bootstrap version (1000 replications, $\mathrm{P}=0.049$ ) of the $\mathrm{Q}$ statistic," even though they did not address the problem of selection criteria in each paper. Their results are very similar to ours, but no remarks were provided by them about the level of significance they used. Nevertheless, we welcome this confirmation of our results with a different statistical procedure.

As they aptly noted, "despite the 20 years of research since the first report, the relationship between aspirin treatment and a reduced risk of preeclampsia in women considered at high-risk still remains an unresolved issue."
However, we certainly disagree with their conclusion that "further meta-analysis considering language bias or individual patient data meta-analysis ${ }^{7}$ are required," as they provokingly proved that it is possible to evaluate the same data (treated as a black box) by different statistical methods. Therefore, we maintain our conclusion that controlled randomized trials with well defined selection criteria would be the best scientific method to answer this question, as we state: "in further randomized controlled trials evaluating the use of low-dose aspirin, participants should be divided into groups according to parity, risk for preeclampsia, and the presence of any prothrombotic factor or disease."1

\section{REFERENCES}

1. Ruano R, Fontes RS, Zugaib M. Prevention of preeclampsia with lowdose aspirin - a systematic review and meta-analysis of the main randomized controlled trials. Clinics. 2005;60:407-14.

2. Subtil D, Goeusse P, Houfflin-Debarge V, Puech F, Lequien P, Breart G, et al. Essai Regional Aspirine Mere-Enfant (ERASME) Collaborative Group. Randomised comparison of uterine artery Doppler and aspirin (100 mg) with placebo in nulliparous women: the Essai Regional Aspirine Mere-Enfant study (Part 2). BJOG. 2003;110:485-91.

3. Yu CK, Papageorghiou AT, Parra M, Palma Dias R, Nicolaides KH; Fetal Medicine Foundation Second Trimester Screening Group. Randomized controlled trial using low-dose aspirin in the prevention of pre-eclampsia in women with abnormal uterine artery Doppler at 23 weeks' gestation. Ultrasound Obstet Gynecol. 2003;22:233-9.
4. Pereira TV, Rudnicki M, Soler JMP, Krieger JE. Meta-analysis of aspirin for the prevention of preeclampsia: do the main randomized controlled trials support an association between low-dose aspirin and a reduced risk of developing preeclampsia? Clinics. in press.

5. Brown H, Prescott R. Applied Mixed Models in Medicine. New York: Wiley; 2001.

6. Egger M, Davey SG, Schneider M, Minder C. Bias in meta-analysis detected by a simple, graphical test. BMJ. 1997;315:629-34.

7. The PARIS Collaboration. Antiplatelet agents for prevention of preeclampsia and its consequences: a systematic review and individual patient data meta-analysis. BMC Pregnancy Childbirth. 2005;5:7. 